Draft OF JULY 4, 2021

Preprint typeset using $\mathrm{LATEX}_{\mathrm{E}}$ style emulateapj v. 12/16/11

\title{
TOWARDS THE MEASUREMENT OF THE MASS OF ISOLATED NEUTRON STARS - PREDICTION OF FUTURE ASTROMETRIC MICROLENSING EVENTS BY PULSARS
}

\author{
ERAN O. OFEK ${ }^{1}$ \\ Draft of July 4, 2021
}

\begin{abstract}
The mass of single neutron stars (NSs) can be measured using astrometric microlensing events. In such events, the center-of-light motion of a star lensed by a NS will deviate from the expected non-lensed motion and this deviation can be used to measure the mass of the NS. I search for future conjunctions between pulsars, with measured proper motion, and stars in the GAIA-DR2 catalog. I identified one candidate event of a star that will possibly be lensed by a pulsar during the next ten years in which the expected light deflection of the background star will deviate from the non-lensed motion by more than $50 \mu$ as. Given the position and proper motion of PSR J0846 - 3533, it will possibly pass $\sim 0.2^{\prime \prime}$ from a $19.0 \mathrm{G}$ magnitude background star in 2022.9. Further assuming a $1.4 \mathrm{M}_{\odot} \mathrm{NS}_{\text {, }}$ the expected maximum deviation of the background star images from the uniform-rate plus parallax motion will be $91 \mu$ as. This pulsar position has a relatively large uncertainty and therefore additional observations are required in order to verify this event. I briefly discuss the opposite case, in which a pulsar is being lensed by a star. Such events can be used to measure the stellar mass via pulsar timing measurements. I do not find good candidates for such events with predicted variations in the pulsar period derivative $(\dot{P})$, divided by $1 \mathrm{~s}$, exceeding $10^{-20} \mathrm{~s}^{-1}$. Since only about $10 \%$ of the known pulsars have measured proper motions, there is potential for an increase in the number of predicted pulsar lensing events.
\end{abstract}

Subject headings: astrometry — gravitational lensing: micro — stars: neutron

\section{INTRODUCTION}

The mass and population mass-range of Neutron Stars (NSs) are fundamental properties related to their formation and evolution and to the equation of state of nuclear matter. So far, we have only obtained accurate mass measurements for NSs in binary systems (e.g., Kramer \& Stairs 2008). There is some evidence that there is more than one channel to form a NS (e.g., Beniamini \& Piran 2016). Therefore, measuring masses of single NS is of great importance.

Paczyński (1995; 1998), Miralda-Escude (1996), and Gould (2000) have suggested to measure stellar masses via the detection of astrometric microlensing events. In such events, a source is lensed by a stellar mass object in our galaxy, and the center-of-light of the source images will deviate from a uniform-rate proper motion. This deviation could be used to measure the mass of the lensing star. Sahu et al. (1998) and Salim \& Gould (2000) made some predictions for future astrometric microlensing events. Harding et al. (2018) estimated the astrometric microlensing rate for known stellar remnants, and McGill et al. (2018) used the GAIA-TGAS catalog (Lindegren et al. 2016) to make predictions for astrometric microlensing events. Furthermore, Bramich et al. (2018) and Mustill et al. (2018) made some predictions for microlensing events in the next ten and twenty years, respectively, based on the GAIA-DR2 catalog. Finally, Lu et al. (2016) and Kains et al. (2017) presented some onging efforts to measure astrometric microlensing events focusing on identifying single stellar-mass black holes in our galaxy, while Sahu et al. (2017) presented the first

\footnotetext{
${ }^{1}$ Benoziyo Center for Astrophysics, Weizmann Institute of Science, 76100 Rehovot, Israel
}

measurements of a white dwarf mass based on an astrometric microlensing event.

Here, I search for future close angular conjunctions between pulsars with a known proper motion and stars in the GAIA-DR2 catalog (Gaia Collaboration et al. 2016; Gaia Collaboration et al. 2018). I find one candidate event in the nearby future, in which a pulsar will pass with a small angular separation from a background star. The impact parameter of this close angular passage is hundreds of times the Einstein radius of the lens. Therefore, this event will not result in a classical microlensing - i.e., events that have a detectable magnification of the background star. However, this event may produce small astrometric shift in the position of the background star relative to the linear motion at a constant angular speed expected from the proper motion component and the periodic variation expected from the parallax. Future measurements of such astrometric microlenisng events may enable the first mass measurements of a single NS.

In 92 , I describe the search for astrometric microlensing events involving pulsars, and the candidate is listed in 3. The results are discussed in 4 .

\section{THE SEARCH}

I selected all the pulsars listed in the Australia Telescope National Facility (ATNF) pulsar databas\&2 (version 1.58 of date 2018 May 10) that have proper motion measurements. The declination of PSR J1856 - 3754 in the ATNF catalog was erroneous 3 . and I use the correct declination from Walter \& Matthews (1997).

\footnotetext{
2 http://www.atnf.csiro.au/people/pulsar/psrcat/

3 The erroneous declination led to the detection of a spurious event that was listed in an earlier version of this paper.
} 


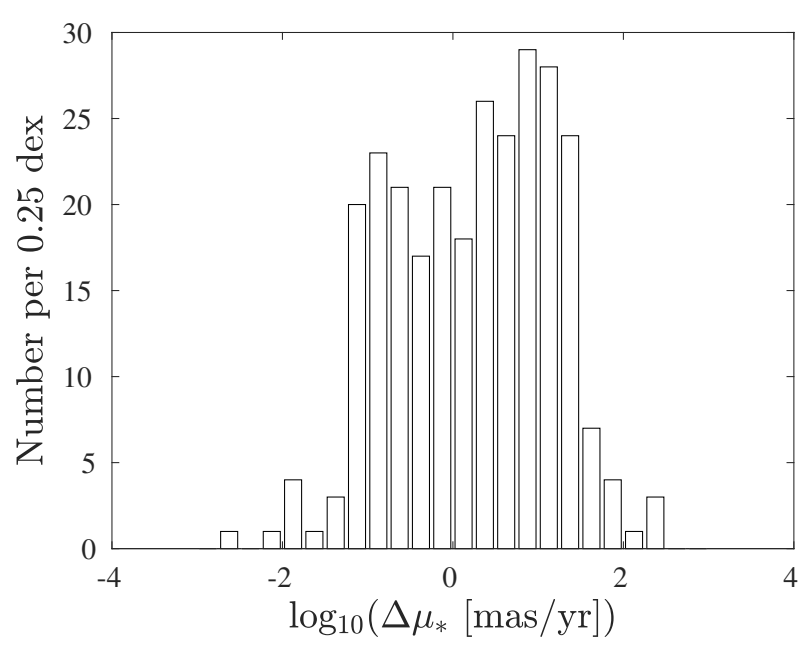

FIG. 1.- The distribution of the errors in the total proper motion of the 277 pulsars with measured proper motions in the ATNF pulsars catalog.

Out of 2636 pulsars in the ATNF catalog, 277 have such measurements. Figure 1 presents the distribution of errors in the proper motions of these pulsars. The typical error in the pulsars proper motion is an order of magnitude larger than that of GAIA-DR2 stars. However, these proper motions are good enough to predict the position of a pulsar to an accuracy of $\sim 0 .^{\prime \prime} 1$ in the next 100 yr.

For each pulsar, I used the catsHTM tool (Soumagnac $\&$ Ofek 2018) to query for all the GAIA-DR2 (Gaia Collaboration et al. 2016; Gaia Collaboration et al. 2018) sources within 1000 arcsec of the pulsar's cataloged position. Given the pulsar's and GAIA-DR2 sources position and proper motion I calculated the closest approach between the stars and the pulsar. For each closest approach with an angular distance below 100 arcsec, that takes place between 2000 and 2100, I calculated the expected astrometric microlensing center-of-light deflection as a function of time. I note that, when the lens distance is small, conjunctions with a larger impact parameter can induce considerable light deflections. However, these deflections will typically change over time scales of decades, making them less attractive for follow up observations.

The light deflection of the center-of-light of the source (i.e., the more distant object of the two) was calculated by taking the positions of the two images of the background star weighted by their respective magnifications, and taking into account the star and pulsar parallaxes. If the star parallax is smaller than two times the parallax error, I set the distance of the star to $10 \mathrm{kpc}$.

In the thin screen, point mass, small angle approximation, the center-of-light of the source light deflection of the images, as measured relative to the source in the direction of the lens, is:

$$
\delta=\frac{\left|m_{+}\right| \theta_{+}+\left|m_{-}\right| \theta_{-}}{\left|m_{+}\right|+\left|m_{-}\right|}-\beta=\frac{\beta / \theta_{E}}{\left(\beta / \theta_{E}\right)^{2}+2} \theta_{E},
$$

where $\beta$ is the angular distance between the lens and source, $\theta_{+}$and $\theta_{-}$are the positions of the two images of the source (relative to the lens), given by (e.g., Einstein

\footnotetext{
${ }^{4}$ Due to the point-mass lens assumption, the third image has infinite demagnification.
}

1936; Liebes 1964; Refsdal 1964; Narayan \& Bartelmann 1996):

$$
\theta_{ \pm}=\frac{1}{2}\left(\beta \pm \sqrt{\beta^{2}+4 \theta_{E}^{2}}\right)
$$

and $m_{+}$and $m_{-}$are the magnifications of the two images

$$
m_{ \pm}=\left[1-\left(\frac{\theta_{E}}{\theta_{ \pm}}\right)^{4}\right]^{-1} .
$$

Here, $\theta_{E}$ is the angular Einstein radius, in radians, given by

$$
\theta_{E}=\sqrt{\frac{4 G M}{c^{2}} \frac{\pi_{\mathrm{rel}}}{A U}},
$$

where $G$ is the gravitational constant, $M$ is the lens mass, $c$ is the speed of light, $A U$ is the astronomical unit, and $\pi_{\text {rel }}$ is the relative parallax in arcseconds

$$
\pi_{\mathrm{rel}}=\frac{\pi_{\mathrm{l}} \pi_{\mathrm{s}}}{\pi_{\mathrm{ls}}}
$$

where $\pi_{l}, \pi_{\mathrm{s}}$, and $\pi_{\mathrm{ls}}$ are the parallaxes between the observer and the lens, the observer and the source, and the lens and the source, respectively. Note that when the images separation $\left(\theta_{+}+\theta_{-}\right)$becomes larger than the instrument resolution, the source deflection angle should not include the demagnified image contribution. In such a case,

$$
\delta=\theta_{+}-\beta
$$

However, for practical purposes, there is no difference between Equation 1 and Equation [6. For the case of $\beta \gg \theta_{\mathrm{E}}$, we can approximate Equation 1 and Equation 6 using

$$
\delta \cong \frac{\theta_{E}^{2}}{\beta}=\frac{4 G M}{c^{2}} \frac{\pi_{1} \pi_{\mathrm{s}}}{\pi_{\mathrm{ls}} A U} \frac{1}{\beta}
$$

Finally, the time scale for the lensing phenomenon is given by $\sim \theta_{\mathrm{E}} / \mu_{*}$, where $\mu_{*}$ is the lens-source relative total proper motion.

Next, I selected sources that have a maximum light deflection above $50 \mu$ as (relative to an event with no microlensing). One obvious contamination is that some pulsars may have companions - if all proper motion measurements are correct, such systems will have constant angular separation between the pulsar and star as a function of time. In order to avoid selecting pulsars with a companion, I selected only sources for which the separation varies by more than $1^{\prime \prime}$ over the $\pm 20 \mathrm{yr}$ of the time of closest approach. The last criterion can be relaxed when cleaner new versions of the GAIA catalog become available.

The search utilized code available as part of the MATLAB astronomy and astrophysics toolbox (Ofek 2014).

\section{CANDIDATE EVENTS}

The selection process described in 92 yielded one candidate for astrometric microlensing events, involving PSR J0846 - 3533. This event will not introduce noticeable magnification of the background source. However, it still can be detected by measuring the position of the background source as a function of time and looking for deviations from the expected linear plus parallax motion.

\footnotetext{
${ }^{5}$ https://webhome.weizmann.ac.il/home/eofek/matlab/
} 
TABLE 1

PSR J0846 - 3533 CONJUNCTION PARAMETERS

\begin{tabular}{ll}
\hline \hline \multicolumn{1}{c}{ Parameter } & \multicolumn{1}{c}{ Value } \\
\hline Pulsar data & PSR J084606.060-353340.64 \\
\hline Name & $08: 46: 06.060 \pm 0.5^{\prime \prime}$ \\
J2000.0 R.A. & $-35: 33: 40.64 \pm 0.5^{\prime \prime}$ \\
J2000.0 Dec. & $-15 \pm 65 \mathrm{mas}$ \\
Proper motion in R.A. & MJD 48719 \\
Proper motion in Dec. & $0.54 \mathrm{kpc}$ \\
Coordinates epoch & $1.1161 \mathrm{~s}$ \\
Distance (Dispersion Measure) & $1.60 \times 10^{-15}$ \\
Period & $1.1 \times 10^{7} \mathrm{yr}$ \\
Period derivative & \\
Characteristic age & $08: 46: 06.291901 \pm 0.15 \mathrm{mas}$ \\
Star data & $-35: 33: 41.34052 \pm 0.18 \mathrm{mas}$ \\
J2000.0 R.A. & $\mathrm{J} 2015.5$ \\
J2000.0 Dec. & $-3.17 \pm 0.32$ \\
Coordinates epoch & $2.80 \pm 0.35$ \\
Proper motion in R.A. & $0.10 \pm 0.23$ \\
Proper motion in Dec. & 0.15 \\
Parallax & $0.38 \mathrm{mas}$ \\
R.A./Dec. correlation & $19.027 \pm 0.002$ \\
Astrometric excess noise & $19.806 \pm 0.048$ \\
Mag G & $18.197 \pm 0.018$ \\
Mag BP & $\mathrm{J} 2022.9$ \\
Mag RP & $\sim 0.22^{\prime \prime}$ \\
\hline Time of minimum separation & $4.5 \mathrm{mas}$ \\
Minimum angular separation & $91 \mu \mathrm{as}$ \\
Estimated Einstein radius & \\
Maximum astrometric deviation & \\
\hline
\end{tabular}

Note. - Due to the large uncertanty in the pulsar coordinates and proper motion, the minimum angular separation, and expected light deflection, are highly uncertain. Pulsar proper motion are adopted from Zou et al. (2005).

This motion can be more complicated if the sources are blended or if it is an astrometric binary.

The details of the event, including the pulsar's parameters, are listed in Table 1. This pulsar have no known companions. Due to the large uncertanty in the pulsar coordinates and proper motion, the minimum angular separation, and expected light deflection, are highly uncertain.

Figures 2 presents the separation and center-of-light deflection as a function of time for PSR J0846 - 3533 . Finding charts of the pulsar field is shown in Figures 3 .

\section{DISCUSSION}

I present a search for conjunctions of pulsars with GAIA-DR2 stars. I find one such possible event, that need to be verified, in which the astrometric deflection, from a constant rate motion of the background star, is expected to be $\sim 0.1$ mas.

This search should be regarded as a preliminary search for candidates. The pulsar's and star's proper motions and distances should be verified with future observations, and it will also be useful to obtain high-resolution imaging of these fields.

An important requirement for such a program is the ability to measure small astrometric shifts, preferably on the level of $1-10 \mu \mathrm{as}$. Current state-of-the-art groundbased observations deliver $100 \mu$ as astrometric precision (e.g., Tendulkar et al. 2012; Lu et al. 2016). Therefore, new techniques and methodologies are required in order to improve the currently available level of precision. I note that the current limitations likely result from systematic errors, and therefore, there is a realistic potential

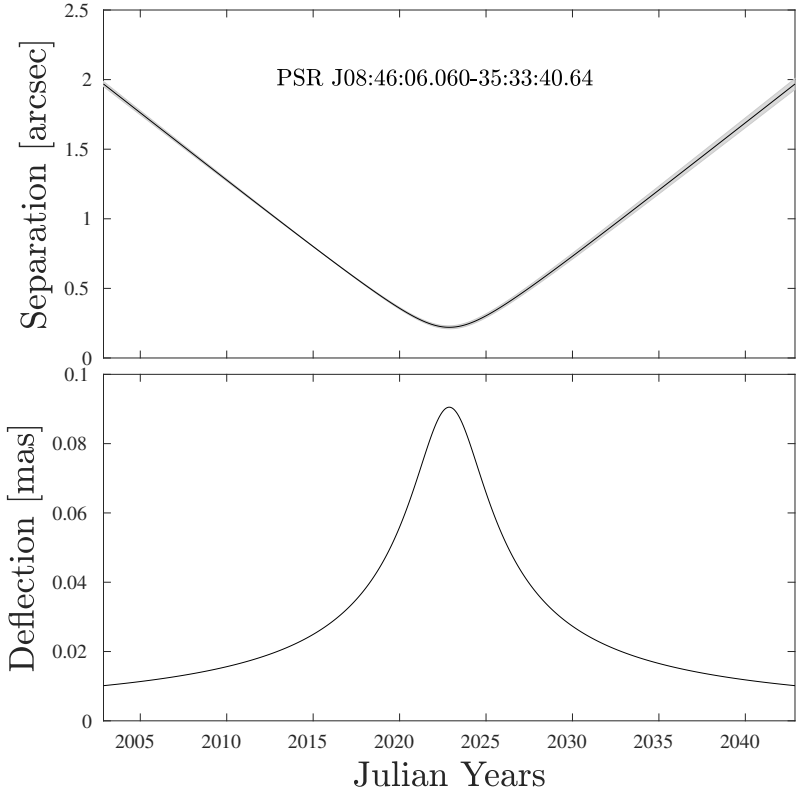

FIG. 2.- PSR J0846 - 3533 angular separation as a function of time from the GAIA star (top panel), and the predicted center-oflight deflection relative to the source position in the direction of the lens (pulsar). The pulsar position is uncertain to about $0.5^{\prime \prime}$ so the minimum separation is still highly uncertain and requires verification.

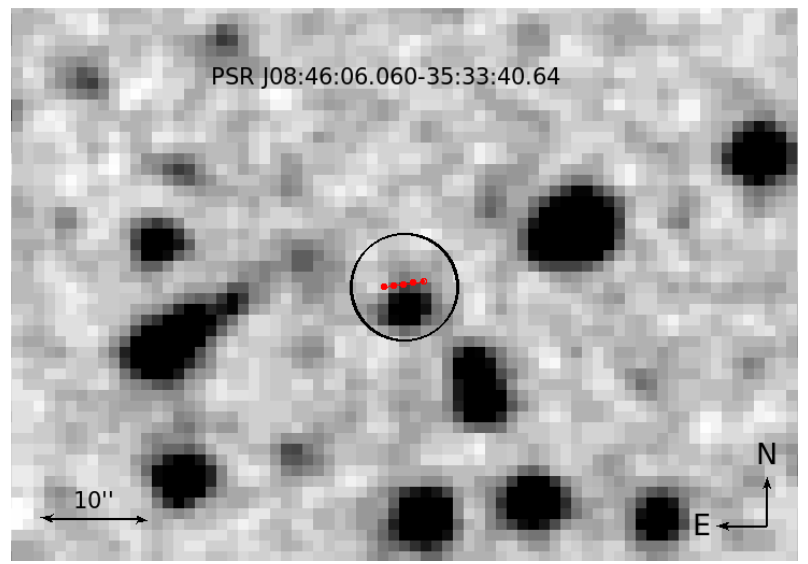

FIG. 3.- Digitized Sky Survey 2 red image of the field of PSR J0846 - 3533. The image gray-scale is inverted. The star's position is marked with a black circle. The red dots mark the pulsar position, every 10 years, from J2002.9 to J2042.9 (East to West).

for improvement.

A related important question is how well do we need to measure the light deflection and distances to the lens and source in order to measure the lens mass to some accuracy (see also Gould 2000). For $\beta \gg \theta_{E}$, the mass depends linearly on $\beta, \delta$ and $1 / \pi_{\text {rel }}$ (Equation 7 ). Specifically, the relative error in the mass estimate will be

$$
\left(\frac{\sigma M}{M}\right)^{2} \propto\left(\frac{\sigma \beta}{\beta}\right)^{2}+\left(\frac{\sigma \delta}{\delta}\right)^{2}+\left(\frac{\sigma \pi_{\mathrm{rel}}}{\pi_{\mathrm{rel}}}\right)^{2} .
$$

Here, $\sigma X$ is the uncertainty in variable $X$. The relative error in the first term $(\beta)$ is expected to be negligible and the relative errors from the second $(\delta)$ and third $\left(\pi_{\text {rel }}\right)$ terms will likely dominate the errors. Therefore, measuring the mass of the NS to better than about $10 \%$ 
accuracy will require controlling the systematic errors in the astrometry to better than $10 \mu \mathrm{as}$, and measuring the relative parallax (which depends on $\pi_{1}$ and $\pi_{\mathrm{s}}$ ) to better than $10 \%$.

Another interesting possibility, already proposed by Larchenkova \& Doroshenko (1995) and Wex et al. (1996), is that a pulsar will be lensed by a star. In principle, this offers the possibility to measure the variations in the lensing time delay via the timing observations of the pulsar. The constant time delay adds a constant phase to the pulsar timing, while the first derivative of the time delay multiplied by the pulsar period adds a constant to the pulsar periodicity. Therefore, these terms cannot be measured. However, the second derivative of the time delay multiplied by the pulsar period adds a constant to the first derivative of the pulsar period $(\dot{P})$, and abrupt variations in $\dot{P}$ (on a year time scale) can be measured. The time delay near a point-mass lens potential drops logarithmically with the impact parameter (see Equation A1) and, therefore, the collective effects of the galactic potential, as well as all the stars near the line of sight, may be important. However, when a star is passing with a small impact parameter (e.g., $\ll 1^{\prime \prime}$ ) it will induce variations in the time delay that are relatively abrupt and that may dominate over all other contributions to the time delay variations. The relevant formulae for the expected time delay are provided, along with an example, in Appendix A. I do not find good candidates for such events of pulsars lensed by foreground stars, which are expected to induce variations in $\dot{P}$, divided by $1 \mathrm{~s}$, exceeding $10^{-20} \mathrm{~s}^{-1}$.

I would like to thank Heng $\mathrm{Xu}$ for pointing out to the erroneous declination of PSR J1856 - 3754 in the ATNF catalog. I am grateful to Andy Gould, Ofer Yaron, and Orly Gnat for comments on the manuscript, to Boaz Katz for useful discussions, and for the support by grants from the Israel Science Foundation, Minerva, Israeli Ministry of Technology and Science, the US-Israel Binational Science Foundation, and the I-CORE Program of the Planning and Budgeting Committee and the Israel Science Foundation.

\section{APPENDIX}

\section{THE SECOND DERIVATIVE OF THE SHAPIRO TIME DELAY}

Larchenkova \& Doroshenko (1995) and Wex et al.(1996) suggested that pulsar time variations can be used to detect an unseen mass or measure the mass of stars.

In the limit that the impact parameter is much larger than the Einstein radius, the geometric time delay can be neglected and the gravitational time delay is given by the Shapiro time delay

$$
\Delta t \cong-\sum_{l} \frac{2 G M_{l}}{c^{3}} \ln \left[1-\cos \left(\beta_{l}\right)\right]
$$

Here, $M_{l}$ is the mass of the $l$-th lens positioned at an angular distance $\beta_{l}$ from the source.

The logarithmic dependence of the time delay on $\beta$ suggests that the stochastic background (e.g., the Galactic potential) is typically the dominant contributor to the time delay. However, when a star is passing with a small impact parameter (e.g., « $1^{\prime \prime}$ ) from a pulsar, then the star gravitational potential may dominate the time delay.

For pulsar observations, the Shapiro time delay induces a phase shift to the pulsar observations. The first derivative of the Shapiro time delay multiplied by the pulsar period, adds a constant to the measured periodicity. The second derivative of the Shapiro time delay, multiplied by the pulsar period, adds a constant to the measured $\dot{P}$. Therefore, we are interested in the second time derivative of the Shapiro time delay, $\ddot{\Delta} t$. For two sources moving with a relative proper motion $\mu_{*}$ (ignoring parallax), the angular distance $\beta$ as a function of time $t^{\prime}$ is

$$
\beta=\sqrt{\beta_{m}^{2}+\left[\mu_{*}\left(t^{\prime}-t_{0}\right)\right]^{2}},
$$

where $t_{0}$ is the time of minimum separation, and $\beta_{m}$ is the minimum impact parameter. Denoting $t=t^{\prime}-t_{0}$, we get

$$
\ddot{\Delta} t=-\frac{G M}{c^{3}} \frac{\mu_{*}^{2}\left(\beta_{m}^{2} \sin \left(\sqrt{t^{2} \mu_{*}^{2}+\beta_{m}^{2}}\right)-t^{2} \mu_{*}^{2} \sqrt{t^{2} \mu_{*}^{2}+\beta_{m}^{2}}\right)}{\left(t^{2} \mu_{*}^{2}+\beta_{m}^{2}\right)^{3 / 2}\left(\cos \left(\sqrt{t^{2} \mu_{*}^{2}+\beta_{m}^{2}}\right)-1\right)} .
$$

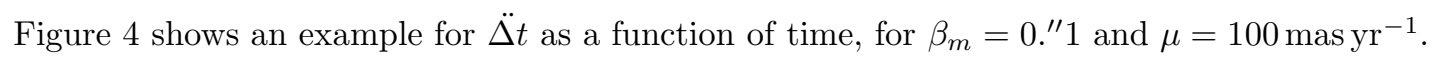

\section{REFERENCES}

Beniamini, P., \& Piran, T. 2016, MNRAS, 456, 4089

Bramich, D. M. 2018, arXiv:1805.10630

Einstein, A. 1936, Science, 84, 506

Gaia Collaboration, Prusti, T., de Bruijne, J. H. J., et al. 2016, A\&A, 595, A1

Gaia Collaboration, Brown, A. G. A., Vallenari, A., et al. 2018, arXiv:1804.09365

Gould, A. 2000, ApJ, 532, 936

Harding, A. J., Stefano, R. D., Lépine, S., et al. 2018, MNRAS, 475,79

Kains, N., Calamida, A., Sahu, K. C., et al. 2017, ApJ, 843, 145

Kramer, M., \& Stairs, I. H. 2008, ARA\&A, 46, 541

Larchenkova, T. I., \& Doroshenko, O. V. 1995, A\&A, 297, 607
Liebes, S. 1964, Physical Review, 133, 835

Lindegren, L., Lammers, U., Bastian, U., et al. 2016, A\&A, 595, A4

Lu, J. R., Sinukoff, E., Ofek, E. O., Udalski, A., \& Kozlowski, S. 2016, ApJ, 830, 41

McGill, P., Smith, L. C., Wyn Evans, N., Belokurov, V., \& Smart, R. L. 2018, MNRAS,

Miralda-Escude, J. 1996, ApJ, 470, L113

Mustill, A. J, Davies, M. B, \& Lindegren, L. 2018, arXiv: 1805.11638

Narayan, R., \& Bartelmann, M. 1996, arXiv:astro-ph/9606001

Ofek, E. O. 2014, Astrophysics Source Ċode Library, ascl: 1407.005 . 


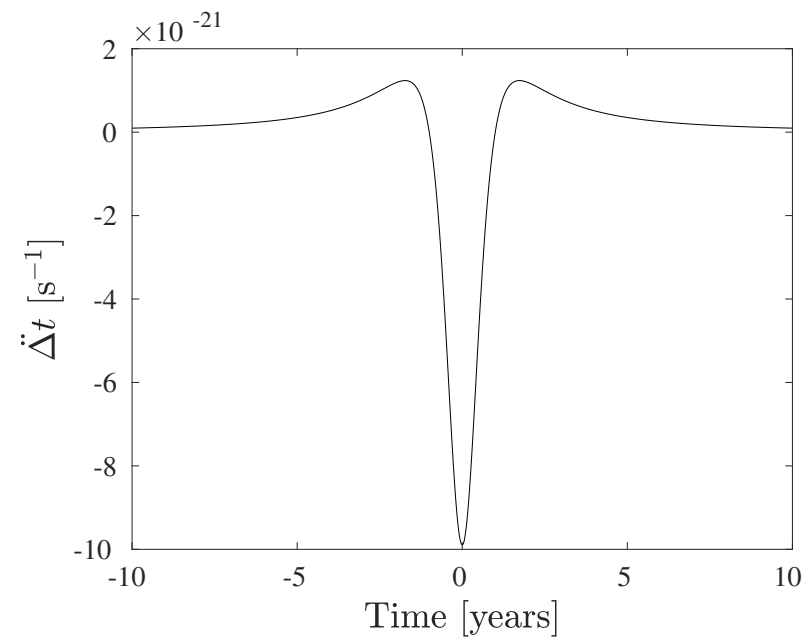

Fig. 4.- The expected $\ddot{\Delta t}$ (neglecting the stochastic background) in the case of a star passing in the foreground of a pulsar with $\beta_{m}=0 .{ }^{\prime \prime} 1$

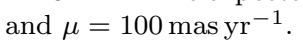

Paczynski, B. 1995, ACTAA, 45, 345

Paczyński, B. 1998, ApJ, 494, L23

Refsdal, S. 1964, MNRAS, 128,295

Sahu, K. C.. Chaney, E. Graham, J., Kane, S., \& Wieldt, D.

1998, Bulletin of the American Astronomical Society, 30, 07.01

Sahu, K. C., Anderson, J., Casertano, S., et al. 2017, Science, 356,1046
Salim, S., \& Gould, A. 2000, ApJ, 539, 241

Soumagnac, M. T., \& Ofek, E. O. 2018, arXiv:1805.02666

Tendulkar, S. P., Cameron, P. B., \& Kulkarni, S. R. 2012, ApJ, 761,76

Walter, F. M., \& Matthews, L. D. 1997, Nature, 390, 315

Wex, N., Gil, J., \& Sendyk, M. 1996, A\&A, 311, 746

Zou, W. Z., Hobbs, G., Wang, N., et al. 2005, MNRAS, 362, 1189 